\title{
Análisis de dos agentes remineralizantes en lesiones incipientes de caries mediante AFM
}

\section{Analysis of two remineralizing agents in incident caries injuries through afm}

Anthony Efraín Moya Fernández

tonnimh@hotmail.com

Universidad Central del Ecuador

Ecuador

https://orcid.org/0000-0003-2570-7048

Tamara Jacqueline Moya Silva

tmoya@gmail.com

Universidad Central del Ecuador

Ecuador

https://orcid.org/0000-0002-6429-8980

María Isabel Zambrano Gutiérrez

reddeinvestigacionrik@fundacionkoinonia.com.ve

Universidad Central del Ecuador

Ecuador

Pablo Mauricio Bonilla Valladares

pablo2us@yahoo.com

Universidad Central del Ecuador

Ecuador

https://orcid.org/0000-0003-1371-1920

Recibido: 25 de abril de 2019

Aprobado: 5 de junio de 2019

\section{RESUMEN}

La caries dental si se trata oportunamente en sus estadios primarios puede ser detenida y limitada con terapias preventivas que favorecen la remineralización. Objetivo: Evaluar la remineralización de lesiones incipientes de caries (mancha blanca) a partir de la aplicación de dos agentes remineralizantes. Materiales y métodos: Se realizó un estudio in - vitro con muestras de esmalte dental divididas en 2 grupos, Grupo A $(n=15)$ y Grupo B $(n=15)$, se generó desmineralización con una solución de ácido láctico y saliva artificial, para la remineralización al Grupo A se le 
aplicó el vidrio bioactivo Fosfosilicato de calcio y sodio fluorado (BAGF), al Grupo B se le aplicó el Fosfopéptido Caseína - fosfato de calcio amorfo fluorado (CPP-ACPF), este procedimiento se lo realizó por 10 días, Resultados: En el Grupo A se obtuvieron valores de $1.967 \mathrm{~nm}, 33.128 \mathrm{~nm}$ y $5.964 \mathrm{~nm}$ respectivamente, en el Grupo B los valores fueron de $2.296 \mathrm{~nm}, 34.335 \mathrm{~nm}$ y $13,611 \mathrm{~nm}$, mediante la prueba estadística t de Student se obtuvo un $p$-valor $=0.108>0.05$ para esmalte sano y un $p$-valor $=0.193$ $>0.05$ para esmalte desmineralizado, lo que indica que no existió una diferencia estadística significativa entre los valores de esmalte sano y desmineralizado de los dos grupos; sin embargo para esmalte remineralizado indicó una gran diferencia entre los valores de remineralización de ambos grupos. Conclusión: Los agentes remineralizantes fosfosilicato de calcio y sodio fluorado (BAGF) y caseína - fosfato de calcio amorfo fluorado (CPP - ACPF) remineralizan el esmalte dental desmineralizado, siendo más efectivo el vidrio bioactivo (BAGF).

Descriptors: Vidrio bioactivo; Caseína; Rugosidad.

\section{SUMMARY}

Dental caries if treated early in their primary stages can be stopped and limited with preventive therapies that favor remineralization. Objective: To evaluate the remineralization of incipient caries lesions (white spot) from the application of two remineralizing agents. Materials and methods: An in-vitro study was carried out with samples of dental enamel divided into 2 groups, Group A $(n=15)$ and Group B $(n=$ 15), demineralization was generated with a solution of lactic acid and artificial saliva, For remineralization, Group A was applied the bioactive glass Calcium fluorinated sodium phosphosilicate (BAGF), Group B was applied the Casein Phosphopeptide fluorinated amorphous calcium phosphate (CPP-ACPF), this procedure was performed by 10 days, Results: In Group A, values of 1,967 nm, 33,128 nm and 5,964 $\mathrm{nm}$ respectively were obtained, in Group B the values were 2,296 nm, 34,335 nm and $13,611 \mathrm{~nm}$, using the Student's t-test, a $p$-value $=0.108>0.05$ for healthy enamel and a $p$-value $=0.193>0.05$ for demineralized enamel, indicating that there was no significant statistical difference between the values of healthy and demineralized enamel of the two groups; however, for remineralized enamel, it indicated a large difference between the remineralization values of both groups. Conclusion: The fluorinated sodium and calcium phosphosilicate remineralizing agents (BAGF) and fluorinated amorphous calcium casein phosphate (CPP - ACPF) remineralize demineralized dental enamel, bioactive glass (BAGF) being more effective.

Descriptors: Bioactive glass, Casein, Roughness 


\section{INTRODUCCIÓN}

En la actualidad, la caries dental se define como una desmineralización del tejido dental, resultado del desequilibrio en las fluctuaciones de $\mathrm{pH}$ entre la biopelícula y el diente. En sus primeras fases es subclínica, pero si la disminución en el pH se perpetúa, la caries dental se hace clínicamente visible, inicialmente en forma de lesión de mancha blanca. Estos conceptos actuales sobre la caries han obligado a los profesionales a usar nuevos sistemas de diagnóstico integral y de manejo de caries dental que les permitan reconocer en los pacientes la presencia de lesiones iniciales o lesiones subclínicas relacionadas con alto riesgo de presentar caries dental, así como adoptar tratamientos no operatorios para interrumpir tempranamente la pérdida de minerales en el tejido dental ${ }^{1}$.

Durante la última década, ha existido una tendencia notable hacia la terapia dental conservadora y hacia la intervención mínima, dejando atrás los conceptos invasivos y meramente restauradores, la mayoría de los investigadores y profesionales del área de odontología consideran grandemente la necesidad de innovar nuevos métodos y estrategias que ayuden a intervenir en forma temprana el proceso de la caries ${ }^{2}$. Dependiendo de los factores de riesgo del paciente, se pueden aplicar una serie de agentes y terapias ${ }^{3}$ para mejorar de manera artificial el proceso de remineralización dentaria y así revertir los daños iniciales producidos por la caries a la estructura dental ${ }^{4}$. La intervención no invasiva de la caries dental se adapta a un criterio en el que las lesiones a nivel del esmalte pueden ser controladas y revertidas ${ }^{5}$ mediante pastas dentales remineralizantes que detengan la progresión de la lesión y promuevan su remineralización antes de que ocurra la cavitación ${ }^{6}$.

El tratamiento no invasivo de las lesiones de caries tempranas por remineralización tiene el potencial de ser un avance importante en el tratamiento clínico de la enfermedad. La mejor estrategia para la gestión de caries es enfocarse en los métodos para mejorar el proceso de remineralización con la ayuda de varios productos remineralizantes ${ }^{7}$. Un producto que puede prevenir la progresión de una lesión de caries y proporcionar remineralización neta en un período de tiempo prolongado, podría provocar un efecto clínico beneficioso significativo, si hay una necesidad 
reducida de intervención. Muchos agentes de remineralización han sido diseñados para potenciar el fluoruro elevando las concentraciones orales de calcio y fosfato, ya sea suministrándolos directamente en lesiones del esmalte 0 indirectamente aumentando sus niveles en placa y saliva, o ambos ${ }^{8}$.

\section{MATERIALES Y MÉTODOS}

Se realizó un estudio de tipo in - vitro, experimental comparativo, con la finalidad de evaluar la remineralización de lesiones incipientes de caries (mancha blanca) a partir de la aplicación de dos agentes remineralizantes ; evaluado y aprobado por el Subcomité de Ética de Investigación en Seres Humanos de la Universidad Central del Ecuador SEISH-UCE. Fueron recolectados 30 dientes premolares humanos, libres de lesiones cariosas, sin restauraciones o previo tratamiento de conductos, sin presencia de fractura coronaria, sin pigmentaciones intrínsecas o extrínsecas, sin fisuras de esmalte o previos desgastes patológicos. Los dientes recibieron una limpieza con curetas periodontales \# $3-4$ y profilaxis con cepillos profilácticos y piedra pómez. Cada diente se cortó con discos de diamante a nivel de la unión amelocementaria, luego se seccionaron las coronas en sentido mesio - distal separando las dos cúspides, se seleccionó la cúspide dentaria más alta (vestibular), las muestras de esmalte con dimensiones de $(4 \mathrm{~mm} \times 5 \mathrm{~mm} \times 2 \mathrm{~mm})$ fueron incrustadas en moldes prefabricados cilíndricos de resina acrílica, asegurando que la superficie vestibular se mantenga expuesta, como lo traza Gokce ${ }^{9}$. Las muestras de esmalte fueron divididas aleatoriamente en dos grupos: Grupo A: fosfosilicato de calcio y sodio fluorado ( $n=15)$ y Grupo B: caseína fosfato de calcio amorfo fluorado $(n=15)$.

Siguiendo el protocolo de Caicedo ${ }^{10} \mathrm{se}$ prepararon $112 \mathrm{ml}$ de solución desmineralizante con $96 \mathrm{ml}$ de Saliva artificial (Salivsol®) y $16 \mathrm{ml}$ de Ácido láctico al $85 \%$, mediante el pH metro (WTW inoLab ph - 720), se verificó un pH2. Cada muestra de esmalte se sumergió en $3.5 \mathrm{ml}$ de solución por 50 min hasta constatar la presencia de caries de mancha blanca.

A las muestras del grupo A, con una jeringa de 1cc se les colocó una delgada capa $(0.5 \mathrm{gr})$ de fosfosilicato de calcio y sodio fluorado y con un microaplicador (Microbrush®) se dispersó en la superficie de examinación, de la misma forma a las 
muestras de grupo B se les colocó una delgada capa (0.5gr) de caseína fosfato de calcio amorfo fluorado. Se almacenaron en envases esterilizados de plástico con saliva artificial donde permanecieron por 24 horas, a $37^{\circ} \mathrm{C}$, en una estufa (Memmert@). La solución de saliva artificial (Salivsol®) fue cambiada a diario. Este proceso se realizó durante 10 días.

Para evaluar la remineralización, las muestras fueron examinadas mediante el Microscopio de fuerza atómica (AFM) Park Systems y el software (Smart ScanTM) ajustado para la obtención de parámetros de rugosidad media $(\mathrm{Ra})$ e imágenes $\mathrm{AFM}$ en tercera dimensión. Se escaneó un área del tercio incisal, medio y cervical de la misma muestra en los tres tiempos de experimentación: en esmalte sano, desmineralizado y remineralizado. Se obtuvieron tres imágenes diferentes, con tres medidas de rugosidad distintas, en una única muestra de estudio y en cada momento de la experimentación. Se determinó el parámetro topográfico Rugosidad media (Ra), debido a que establece un valor cuantitativo medio a partir de los distintos niveles de altura que presentan los picos de las superficies escaneadas.

\section{RESULTADOS}

Se reflejan los valores de rugosidad ( $\mathrm{Ra}$ ) obtenidos en las muestras sometidas a las pruebas con BAGF y CPP-ACPF en la región dental incisal, media y cervical de las muestras analizadas. Se observa que de un valor muy bajo de rugosidad (Ra) en el esmalte sano, después de la desmineralización la rugosidad (Ra) se incrementa ostensiblemente y luego de la remineralización el valor de rugosidad (Ra) obtenido es nuevamente bajo, lo cual da como resultado una variación entre el resultado inicial y final. (Tabla 1, Tabla 2) 
Pruebas con Fosfosilicato de calcio y sodio fluorado (Grupo A) _ Promedio.

Esmalte sano Desmineralizado Remineralizado

$\mathrm{Ra}(\mathrm{nm})$ $\mathrm{Ra}(\mathrm{nm})$

$\mathrm{Ra}(\mathrm{nm})$

VARIACIÓN

\section{Región Incisal}
1.967
33.128
5.964
3.997

Región Media
2.219
35.008
6.391
4.172

\section{Región Cervical}
1.811
34.118
6.340
4.529

Valores de rugosidad de esmalte sano desmineralizado y remineralizado Grupo B.

Tabla 2.

Pruebas con caseína fosfato de calcio amorfo fluorado (Grupo B) Promedio.

\begin{tabular}{|c|c|c|c|}
\hline $\begin{array}{l}\text { Esmalte sano } \\
\qquad \mathrm{Ra}(\mathrm{nm})\end{array}$ & $\begin{array}{c}\text { Desmineralizado } \\
\operatorname{Ra}(\mathbf{n m})\end{array}$ & $\begin{array}{c}\text { Remineralizado } \\
\qquad \mathbf{R a}(\mathrm{nm})\end{array}$ & VARIACIÓN \\
\hline \multicolumn{4}{|c|}{ Región Incisal } \\
\hline 2.504 & 36.076 & 13.920 & 11.416 \\
\hline \multicolumn{4}{|c|}{ Región Media } \\
\hline 2.347 & 34.585 & 13.631 & 11.284 \\
\hline \multicolumn{4}{|c|}{ Región Cervical } \\
\hline 2.296 & 34.335 & 13.611 & 11.315 \\
\hline
\end{tabular}


En los Estadígrafos de medición para la prueba t de Student se describe la media obtenida en los dos grupos de la muestra (15 unidades cada una), y de acuerdo con la desviación estándar se muestra la dispersión entre los valores de rugosidad (Ra) del esmalte sano, desmineralizado y remineralizado de ambos grupos. Los resultados de la prueba estadística de t de Student presentan un p-valor $=0.108>0.05(5 \%$ de error permitido) para la relación de las muestras de esmalte sano, mostrando que no existe diferencia significativa entre ellos, luego se obtiene un $p$-valor $=0.193>0.05$ ( $5 \%$ de error permitido) para las muestras de esmalte desmineralizado, mostrando también que estos resultados no son estadísticamente significativos. (Tabla 3, Tabla $4)$.

Desviación estándar para esmalte sano desmineralizado y remineralizado de ambos Grupos.

Tabla 3

\begin{tabular}{|c|c|c|c|c|}
\hline Pares & Variables comparadas & Media & $\mathbf{N}$ & $\begin{array}{l}\text { Desviación } \\
\text { estándar }\end{array}$ \\
\hline \multicolumn{5}{|c|}{ Región Incisal } \\
\hline \multirow{3}{*}{ Par 1} & Esmalte sano - BAGF. & 1.9668 & 15 & 0.90829 \\
\hline & Esmalte sano - CPP-ACPF. & 2.5044 & 15 & 1.17777 \\
\hline & Desmineralizado - BAGF. & 33.1279 & 15 & 4.68486 \\
\hline Par 2 & $\begin{array}{c}\text { Desmineralizado - CPP- } \\
\text { ACPF. }\end{array}$ & 36.0763 & 15 & 7.73965 \\
\hline \multirow[b]{2}{*}{ Par 3} & Remineralizado - BAGF. & 5.9639 & 15 & 0.90616 \\
\hline & Remineralizado - CPP-ACPF. & 13.9205 & 15 & 2.78616 \\
\hline \multicolumn{5}{|c|}{ Región Media } \\
\hline \multirow{3}{*}{ Par 1} & Esmalte sano - BAGF. & 2.2193 & 15 & 1.10440 \\
\hline & Esmalte sano - CPP-ACPF. & 2.3471 & 15 & 1.17812 \\
\hline & Desmineralizado - BAGF. & 35.0077 & 15 & 6.00242 \\
\hline
\end{tabular}

Prueba t de Student para esmalte sano desmineralizado y remineralizado entre ambos Grupos. Tabla 4 


\begin{tabular}{|c|c|c|c|c|}
\hline Par 2 & Desmineralizado - CPP- & \multirow{2}{*}{$\mathbf{T}^{15}$} & \multicolumn{2}{|c|}{7.14051} \\
\hline Pares & Variables comparadas & & gl & $\begin{array}{c}p- \\
\text { valor }\end{array}$ \\
\hline Par 3 & Remineralizado - CPRerơfFlncisal 3.63 & 15 & \multicolumn{2}{|c|}{2.71476} \\
\hline Par 1 & $\begin{array}{c}\text { Esmalte sano BAGF - Esmalte sano } \\
\text { CPP-ACPF. }\end{array}$ & -1.715 & 14 & 0.108 \\
\hline Par 1 & EsmaDessmimeraltzaredoABAGF - & 15 & \multicolumn{2}{|c|}{0.88268} \\
\hline Par 2 & 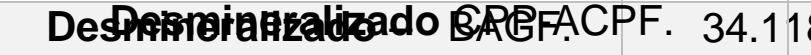 & -1.365 & \multicolumn{2}{|c|}{719785693} \\
\hline \multirow[t]{2}{*}{ Par 3} & $\begin{array}{c}\text { Remineralizado BAGF - } \\
\text { Remineralizado CPP-ACPF. }\end{array}$ & -11.048 & 14 & 0.000 \\
\hline & Remineralizado - BReglón Media6.339 & 15 & \multicolumn{2}{|c|}{1.51968} \\
\hline Par 1 & $\begin{array}{c}\text { Esmalte sano BAGF - Esmalte sano } \\
\text { CPP-ACPF. }\end{array}$ & -0.270 & 14 & 0.791 \\
\hline Par 2 & $\begin{array}{l}\text { Desmineralizado BAGF - } \\
\text { Desmineralizado CPP-ACPF. }\end{array}$ & -0.174 & 14 & 0.865 \\
\hline Par 3 & $\begin{array}{c}\text { Remineralizado BAGF - } \\
\text { Remineralizado CPP-ACPF. }\end{array}$ & -9.313 & 14 & 0.000 \\
\hline \multicolumn{5}{|c|}{ Región Cervical } \\
\hline Par 1 & $\begin{array}{c}\text { Esmalte sano BAGF - Esmalte sano } \\
\text { CPP-ACPF. }\end{array}$ & -1.222 & 14 & 0.242 \\
\hline Par 2 & $\begin{array}{l}\text { Desmineralizado BAGF - } \\
\text { Desmineralizado CPP-ACPF. }\end{array}$ & -0.084 & 14 & 0.935 \\
\hline Par 3 & $\begin{array}{c}\text { Remineralizado BAGF - } \\
\text { Remineralizado CPP-ACPF. }\end{array}$ & -9.700 & 14 & 0.000 \\
\hline
\end{tabular}

observa en la tabla 5 que el p-valor $=0.000<0.05$ ( $5 \%$ de error permitido) para las muestras en esmalte remineralizado, indican que el resultado de rugosidad (Ra) con BAGF es totalmente diferente al obtenido con CPP - ACPF. La relación entre la rugosidad $(\mathrm{Ra})$ de las muestras en esmalte sano y remineralizado con BAGF y CPPACPF, reflejó un $p$-valor $=0.000<0.05$ (5\% de error permitido), mostrando una 
diferencia significativa entre la rugosidad $(\mathrm{Ra})$ de la muestra de esmalte dental sano y luego de haber sido remineralizado.

Prueba t de Student para esmalte sano y remineralizado de ambos Grupos.

Tabla 5

Prueba t de Student para comparar los valores de la prueba al inicio y final del experimento por cada compuesto

\begin{tabular}{|c|c|c|c|c|}
\hline Pares & Variables comparadas & $t$ & gl & $p$-valor \\
\hline \multicolumn{5}{|c|}{ Región Incisal } \\
\hline Par 1 & $\begin{array}{c}\text { Esmalte sano BAGF - Remineralizado } \\
\text { CPP-ACPF. }\end{array}$ & 31.785 & 14 & 0.000 \\
\hline Par 2 & $\begin{array}{c}\text { Esmalte sano BAGF - Remineralizado } \\
\text { CPP-ACPF. }\end{array}$ & $\begin{array}{c}- \\
27.193\end{array}$ & 14 & 0.000 \\
\hline \multicolumn{5}{|c|}{ Región Media } \\
\hline Par 1 & $\begin{array}{c}\text { Esmalte sano BAGF - Remineralizado } \\
\text { CPP-ACPF. }\end{array}$ & 20.709 & 14 & 0.000 \\
\hline Par 2 & $\begin{array}{l}\text { Esmalte sano CPP-ACPF. - } \\
\text { Remineralizado CPP-ACPF. }\end{array}$ & $\begin{array}{c}- \\
28.209\end{array}$ & 14 & 0.000 \\
\hline \multicolumn{5}{|c|}{ Región Cervical } \\
\hline Par 1 & $\begin{array}{l}\text { Esmalte sano BAGF - Remineralizado } \\
\text { BAGF. }\end{array}$ & 31.785 & 14 & .000 \\
\hline Par 2 & $\begin{array}{c}\text { Esmalte sano CPP-ACPF - Remineralizado } \\
\text { CPP-ACPF. }\end{array}$ & $\begin{array}{c}- \\
27.193\end{array}$ & 14 & .000 \\
\hline
\end{tabular}

\section{DISCUSIÓN}

En la actualidad la odontología preventiva ha establecido en la investigación una opción terapéutica adecuada para el manejo de las lesiones incipientes de caries (mancha blanca), que integra la prevención, la remineralización y los procedimientos 
mínimamente invasivos en un nuevo paradigma de cuidados de la salud oral ${ }^{11}$. Los enfoques no operatorios, diseñados para evitar la progresión de las lesiones cariosas del esmalte se han relacionado con el uso de pastas dentales remineralizantes, encaminando el manejo clínico de la enfermedad hacia un esquema integral de atención en salud oral primaria ${ }^{12}$.

Rehder Neto, F. ${ }^{13}$. en un estudio realizado en 75 especímenes de esmalte dental tratados con agentes remineralizantes, encontró que los dentífricos fluorados a base de calcio y fosfato podrían ser útiles para controlar la progresión de lesiones iniciales de caries, Así mismo Diamanti, I. ${ }^{14}$ realizó pruebas de remineralización en muestras de esmalte con pastas dentífricas a tres concentraciones diferentes de fluoruro de sodio y un sistema de fosfosilicato de calcio y sodio, obteniendo como resultado que estos compuestos inhiben la desmineralización y promueven la remineralización de forma efectiva, no obstante el fosfosilicato de calcio y sodio fluorado y el caseína fosfato de calcio amorfo fluorado en 10 días de experimentación in - vitro mostraron efecto remineralizante.

Palaniswamyet, U. ${ }^{15}$ comparó el potencial de remineralización del vidrio bioactivo (BAG) y el fosfopéptido de caseína fosfato de calcio amorfo (CPP-ACP) en lesiones tempranas de caries, concluyendo que el vidrio bioactivo es más eficaz inicialmente pero que ambos materiales pueden ser utilizados para revertir el proceso inicial de la caries. Este efecto reparativo también fue observado para fosfosilicato de calcio y sodio fluorado, ya que se mostró más activo a la caseína - fosfato de calcio amorfo fluorado en la remineralización.

En nuestros días concurre un consenso amplio sobre las posibilidades de hacer tratamientos a las lesiones de caries en sus diferentes momentos de evolución. En este panorama, las estrategias y los productos remineralizantes se convierten en parte del arsenal de los servicios de salud y los odontólogos para impactar en menor tiempo en los índices de incidencia y prevalencia de caries; por ello, conocerlos y estudiarlos debe ser una prioridad para las facultades de odontología, con el fin de integrar su uso a las herramientas de tratamiento que los odontólogos puedan ofrecer ${ }^{1}$. 
Se concluye que los valores de rugosidad demostraron que los agentes remineralizantes fosfosilicato de calcio y sodio fluorado (BAGF) y caseína - fosfato de calcio amorfo fluorado (CPP - ACPF) remineralizan el esmalte dental desmineralizado, siendo más efectivo el vidrio bioactivo (BAGF).

\section{REFERENCIAS CONSULTADAS}

1. Castellanos JE, Marín LM, Úsuga MV, Castiblanco GA, Martignon S. La remineralización del esmalte bajo el entendimiento actual de la caries dental. Univ Odontol. 2013; 32(69): 49-59.

2. Cummins D. Caries dental: Una enfermedad que, en el siglo XXI, continua siendo un motivo de inquietud en relación con la salud pública: la exploración de una tecnología innovadora para la prevención de la caries. The Journal of Clinical Dentistry. 2013; 24: p. A10.

3. Vargas $\mathrm{J}$, Vargas del Valle $\mathrm{P}$, Palomino $\mathrm{H}$. Lesiones de mancha blanca en Ortodoncia: Conceptos actuales. Avances en Odontoestomatología. 2016; 32(4): p. 218.

4. González C, Garrocho A, Pérez F, Amaury P. Eficacia de tres tratamientos para la remineralización de la lesión incipiente de caries o mancha blanca en pacientes con ortodoncia. Revista Mexicana de Odontología Clínica; IntraMed. 2009 Nov; 2(14): p. 3

5. López M, Cástro J. La terapia remineralizadora en la práctica preventiva y restauradora de la odontología. ResearchGate. Jan. 2008; 10(11): p. 22- 31.

6. Thepyou R, et a. Casein phosphopeptide-amorphous calcium phosphate and glass ionomer show distinct effects in the remineralization of proximal artificial caries lesion in situ. Dental Materials Journal. 2013 Feb; 4(32): p. p. 648.

7. Chaudhary I, Tripathi A, Yadav G, Saha S. Effect of Casein Phosphopeptideamorphous Calcium Phosphate and Calcium Sodium Phosphosilicate on Artificial Carious Lesions: An in vitro Study. Int J Clin Pediatr Dent. 2017; 10(3): p. 261.

8. Lynch RJ, Smith SR. Remineralization Agents - New and Effective or Just Marketing Hype? SAGE. 2012 Ago; 24(2): p. 64 - 65. 
9. Gokce G, Savas S, Kucukyilmaz E, Veli I. Effects of toothpastes on white spot lesions around orthodontic brackets using quantitative light-induced fluorescence (QLF). J Orofac Orthop. 2017 ; 78(6): p. 482.

10. Chicaiza G, Navarrete N. Efecto de dos agentes remineralizantes en lesiones de mancha blanca: Estudio in Vitro. Revista "Odontología". 2016 ; 18(1): p. 7 8.

11. Jingarwar M, Bajwa, Pathak A. Minimal Intervention Dentistry - A New Frontier in Clinical Dentistry. Journal of Clinical and Diagnostic Research: JCDR. 2014 Jul; 8(7): p. 4.

12. Frencken J. Atraumatic restorative treatment and minimal intervention dentistry. Official journal of the British Dental Association. 2017 Aug; 223(3): p. 183.

13. Rehder F, Maeda F, Turssi C, Serra M. Potential agents to control enamel caries-like lesions. journal of dentistry ELSEVIER. 2009 Oct; 37(10): p. 786789.

14. Diamanti I, Koletsi-Kounari H, Mamai-Homata E, Vougiouklakis G. In vitro evaluation of fluoride and calcium sodium phosphosilicate toothpastes, on root dentine caries lesions. Journal of dentistry ELSEVIER. 2011 Sep; 39(9): p. 619628.

15. Palaniswamy U, Prashar N, Kaushik M, Lakkam S, Arya S, Pebbeti S. A comparative evaluation of remineralizing ability of bioactive glass and amorphous calcium phosphate casein phosphopeptide on early enamel lesion. Dental Research Journal. 2016; 13(4): p. 297-302.

\section{REFERENCES CONSULTED}

1. Castellanos JE, Marín LM, Úsuga MV, Castiblanco GA, Martignon S. The remineralization of enamel under the current understanding of tooth decay. Univ Odontol. 2013; 32 (69): 49-59.

2. Cummins D. Dental caries: A disease that, in the 21st century, continues to be a cause for concern in relation to public health: the exploration of an innovative technology for caries prevention. The Journal of Clinical Dentistry. 2013; 24: p. A10 
3. Vargas $\mathrm{J}$, Vargas del Valle P, Palomino H. White spot lesions in Orthodontics: Current concepts. Advances in Odontoestomatology. 2016; 32 (4): p. 218.

4. González C, Garrocho A, Pérez F, Amaury P. Efficacy of three treatments for remineralization of incipient caries lesion or white spot in patients with orthodontics. Mexican Journal of Clinical Dentistry; IntraMed. 2009 Nov; 2 (14): p. 3

5. López M, Cástro J. Remineralizing therapy in the preventive and restorative practice of dentistry. ResearchGate Jan. 2008; 10 (11): p. 22-31.

6. Thepyou R, et a. Casein phosphopeptide-amorphous calcium phosphate and glass ionomer show distinct effects in the remineralization of proximal artificial caries lesion in situ. Dental Materials Journal 2013 Feb; 4 (32): p. p. 648.

7. Chaudhary I, Tripathi A, Yadav G, Saha S. Effect of Casein Phosphopeptideamorphous Calcium Phosphate and Calcium Sodium Phosphosilicate on Artificial Carious Lesions: An in vitro Study. Int J Clin Pediatr Dent. 2017; 10 (3): p. 261

8. Lynch RJ, Smith SR. Remineralization Agents - New and Effective or Just Marketing Hype? SAGE 2012 Aug; 24 (2): p. 64-65.

9. Gokce G, Savas S, Kucukyilmaz E, Veli I. Effects of toothpastes on white spot lesions around orthodontic brackets using quantitative light-induced fluorescence (QLF). J Orofac Orthop. 2017; 78 (6): p. 482

10. Chicaiza G, Navarrete N. Effect of two remineralizing agents on white spot lesions: In Vitro Study. "Dentistry" magazine. 2016; 18 (1): p. 7-8.

11. Jingarwar M, Bajwa, Pathak A. Minimal Intervention Dentistry - A New Frontier in Clinical Dentistry. Journal of Clinical and Diagnostic Research: JCDR. 2014 Jul; 8 (7): p. Four.

12. Frencken J. Atraumatic restorative treatment and minimal intervention dentistry. Official journal of the British Dental Association. 2017 Aug; 223 (3): p. 183

13. Rehder F, Maeda F, Turssi C, Serra M. Potential agents to control enamel caries-like lesions. Journal of Dentistry ELSEVIER. 2009 Oct; 37 (10): p. $786-$ 789.

14. Diamanti I, Koletsi-Kounari H, Mamai-Homata E, Vougiouklakis G. In vitro evaluation of fluoride and calcium sodium phosphosilicate toothpastes, on root dentine caries lesions. Journal of dentistry ELSEVIER. 2011 Sep; 39 (9): p. 619628. 
15. Palaniswamy U, Prashar N, Kaushik M, Lakkam S, Arya S, Pebbeti S. A comparative evaluation of remineralizing ability of bioactive glass and amorphous calcium phosphate casein phosphopeptide on early enamel lesion. Dental Research Journal 2016; 13 (4): p. 297-302.

(C2020 por los autores. Este artículo es de acceso abierto y distribuido según los términos y condiciones de la licencia Creative Commons Atribución-NoComercial-Compartirlgual 4.0 Internacional (CC BY-NC-SA 4.0) (https://creativecommons.org/licenses/by-nc-sa/4.0/). 